This item was submitted to Loughborough's Research Repository by the author.

Items in Figshare are protected by copyright, with all rights reserved, unless otherwise indicated.

\title{
Identification of ankle sprain motion from common sporting activities by dorsal foot kinematics data
}

PLEASE CITE THE PUBLISHED VERSION

http://dx.doi.org/10.1016/j.jbiomech.2010.03.014

\section{PUBLISHER}

(C) Elsevier

\section{VERSION}

AM (Accepted Manuscript)

\section{PUBLISHER STATEMENT}

This work is made available according to the conditions of the Creative Commons Attribution-NonCommercialNoDerivatives 4.0 International (CC BY-NC-ND 4.0) licence. Full details of this licence are available at: https://creativecommons.org/licenses/by-nc-nd/4.0/

\section{LICENCE}

CC BY-NC-ND 4.0

\section{REPOSITORY RECORD}

Chan, Yue-Yan, Daniel Tik-Pui Fong, Mandy Man-Ling Chung, Wen-Jung Li, Wei-Hsin Liao, Patrick Shu-Hang Yung, and Kai-Ming Chan. 2019. "Identification of Ankle Sprain Motion from Common Sporting Activities by Dorsal Foot Kinematics Data”. figshare. https://hdl.handle.net/2134/21275. 


\section{Elsevier Editorial System(tm) for Journal of Biomechanics Manuscript Draft}

Manuscript Number: BM-D-09-01010R1

Title: Identification of ankle sprain motion from common sporting activities by dorsal foot kinematics data

Article Type: Full Length Article (max 3000 words)

Keywords: Gyrometer, accelerometer, motion sensor, Support Vector Machine, injury prevention, injury mechanism

Corresponding Author: Dr Daniel Tik-Pui Fong, PhD

Corresponding Author's Institution: The Chinese University of Hong Kong

First Author: Yue-Yan Chan, MPhil

Order of Authors: Yue-Yan Chan, MPhil; Daniel Tik-Pui Fong, PhD; Mandy Man-Ling Chung; Wen Jung Li; Wei-Hsin Liao; Kai-Ming Chan 


\section{Cover letter}

Editor of Journal of Biomechanics,

REF: Submission of manuscript "Identification of Ankle Sprain Motion from Normal Activities by Dorsal Foot Kinematics Data"

The authors would like to submit this paper as an "Original Article". We declare that each author were fully involved in the study and preparation of the manuscript and that the material within has not been and will not be submitted for publication elsewhere.

Corresponding author during review process: Ms. Yue-Yan CHAN, Department of Orthopaedics and Traumatology, Prince of Wales Hospital, Faculty of Medicine, The Chinese University of Hong Kong, Hong Kong, China, yychan@ort.cuhk.edu.hk

Corresponding author if paper is accepted: Prof. Daniel Tik-Pui Fong, Department of Orthopaedics and Traumatology, Prince of Wales Hospital, Faculty of Medicine, The Chinese University of Hong Kong, Hong Kong, China, dfong@ort.cuhk.edu.hk

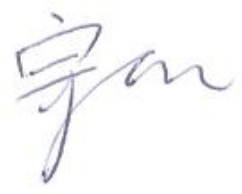

Yue-Yan CHAN

November 4th, 2009. 
Dear Editor,

Here is the specific reply to reviewers, thank you.

Reviewer \#1:

This is a good paper that describes the sprain motion detection system using the foot kinematic signals and the machine learning tool (SVM).

The methodology is well presented, and the identification/classification results are acceptable.

The discussion section is appropriate to explain the incorrect classifications in the supination sprain trials.

There are still some places need to improve:

(1) In Page 4, Paragraph 2, Line 3: the citation (Chan et al. 2008) is a mistake-- I guess it should be (Chan et al. 1998), as the reference list does not include (Chan et al. 2008).

$\rightarrow$ Corrected. The reference should be Chan et al. 2008 which was typed wrongly as 1998 in the reference list.

(2) In Page 7, Paragraph 1, Line 6: it is better to rewrite the symbol for the kernel function "k" as "K(·)".

$\rightarrow$ Corrected as instructed.

(3) In Page 7, Paragraph 1, Line 7: the inline equation "K(x_i, $x)=$

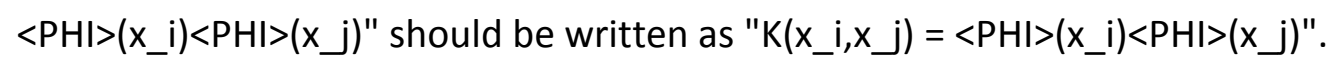
$\rightarrow$ Corrected as instructed.

(4) In Page 9, Section Results, Paragraph 1, Line 5: the decimal fraction of the threshold $b=0.46397071$ could be shorten.

$\rightarrow$ The decimal fraction of the threshold was shorten.

It is better to interpret the physical meaning of such a threshold value in the experiment, and how it affects the classification results (accuracy).

$\rightarrow$ Thank you for the question. The threshold ' $b$ ' is the bias term which does not 
affect the accuracy much if there are enough features. As we have 521 supports vectors in the model, this bias term does not alter much in the accuracy.

(5) The eyes of the subjects in Figure 2 should be masked.

$\rightarrow$ Ammended. The eyes of the subject were masked.

Reviewer \#2:

General comment:

This is a well-written and interesting paper. This study aimed to present an identification system to detect sprain motion. The part of materials and methods and results should be described in more detail, especially how to perform the validation test.

$\rightarrow$ Thank you for your comments. Some sentences were added to the SVM training and validation part for better understanding:

'This process allowed the waveform characteristics being extracted for the training. Only low frequency components were fed into the Support Vector Machine, while high frequency noises were eliminated.'

'The Support Vector Machine then generated the model which consists of a hyperplane to separate the two sets of data: sprain motion and common sporting activities.'

'The same data collection procedure for the aforementioned SVM training session was done. Each subject performed a total of 100 trials: 50 trials of simulated supination sprain motion (Figure 2 ) and 50 trials of non-sprain motion. One second of sensor data from each trial was then trimmed and converted to frequency domain using DFT. Processed data was then fed into the trained SVM model. The accuracy was calculated by the percentage of trials being correctly identified.'

Besides, this article has well organization in the context and clear explanation in discussion and conclusion. It would be helpful to provide more information if the author could take more efforts on description of contribution in current studies and compare the result with other publication. 
$\rightarrow$ Thank you for the suggestion. Reference of Shi et al. 2009 and Bourke et al. 2007 were added. They are similar studies working on identifying fall motion from normal motions using accelerometers. Their accuracy is comparable with our study.

Specific comment:

1.Page 8, Materials and method section. It's suggested to provide more detail information about "Support Vector Machine verification". Author should try to illustrate the relation between SVM and DFT.

$\rightarrow$ Thank you for the suggestion. Some sentences were added to the SVM training and validation part for better understanding, relation between SVM and DFT was also stated in the first sentence:

'This process allowed the waveform characteristics being extracted for the training. Only low frequency components were fed into the Support Vector Machine, while high frequency noises were eliminated.'

'The Support Vector Machine then generated the model which consists of a hyperplane to separate the two sets of data: sprain motion and common sporting activities.'

'The same data collection procedure for the aforementioned SVM training session was done. Each subject performed a total of 100 trials: 50 trials of simulated supination sprain motion (Figure 2) and 50 trials of non-sprain motion. One second of sensor data from each trial was then trimmed and converted to frequency domain using DFT. Processed data was then fed into the trained SVM model. The accuracy was calculated by the percentage of trials being correctly identified.'2.Page 10, Discussion section. Insufficient comparison of the relation between simulated supination sprains and real sprain case. It's the major limitation because that real sprain is not ethical and is not reproducible in laboratory. Therefore, if it can be acted as an alarm to activate the protective mechanism for the intelligent sprain-free shoe system in the future, additional description and verification are necessary.

Discussion on the realtion between simulated supination sprains and real sprain cases were added.

'We believed that those simulated supination sprain motions are less vigorous than the real sprain motions. This means that the simulated sprain motion have 
measured linear acceleration and angular velocity more similar to that of common sporting motions. Thus, we believed that as this classification model can classify simulated sprain motion from common sporting motions in $91.3 \%$ accuracy, the system can work at a similar or even better accuracy in real sprain classification. However, this belief can only be proved by manufacturing prototype which will be applied directly to athletics during training and competitions.'

Thank you very much for your comments.

Best regards, Yue Yan CHAN 
Identification of ankle sprain motion from common sporting activities by dorsal foot kinematics data

Yue-Yan Chan ${ }^{\mathrm{a}, \mathrm{b}, \mathrm{c}}$, Daniel Tik-Pui Fong ${ }^{\mathrm{a}, \mathrm{c}}$, Mandy Man-Ling Chung ${ }^{\mathrm{a}, \mathrm{c}}$, Wen-Jung Li ${ }^{\mathrm{d}}$, Wei-Hsin Liao ${ }^{\text {d }}$, Patrick Shu-Hang Yung ${ }^{\text {a,b,c }}$, Kai-Ming Chan ${ }^{\text {a,c }}$

${ }^{a}$ Department of Orthopaedics and Traumatology, Prince of Wales Hospital, Faculty of Medicine, The Chinese University of Hong Kong, Hong Kong, China

${ }^{\mathrm{b}}$ Department of Orthopaedics and Traumatology, Alice Ho Miu Ling Nethersole Hospital, Hong Kong, China

${ }^{\mathrm{c}}$ The Hong Kong Jockey Club Sports Medicine and Health Sciences Centre, Faculty of Medicine, The Chinese University of Hong Kong, Hong Kong, China

${ }^{\mathrm{d}}$ Department of Mechanical and Automation Engineering, Faculty of Engineering, The Chinese University of Hong Kong, Hong Kong, China

\section{$\underline{\text { Abstract }}$}

This study presented a method to identify ankle sprain motion from common sporting activities by dorsal foot kinematics data. Six male subjects performed 300 simulated supination sprain trials and 300 non-sprain trials in a laboratory. Eight motion sensors were attached to the right dorsal foot to collect three-dimensional linear acceleration and angular velocity kinematics data, which were used to train up a Support Vector Machine (SVM) model for the identification purpose. Results 
suggested that the best identification method required only one motion sensor located at the medial calcaneus, and the method was verified on another group of six subjects performing 300 simulated supination sprain trials and 300 non-sprain trials. The accuracy of this method was $91.3 \%$, and the method could help developing a mobile motion sensor system for ankle sprain detection.

\section{Introduction}

Ankle sprain is one of the most common ankle injuries in sports (Fong et al., 2007), in which human reflex response may not be fast enough to accommodate the sudden explosive motion and prevent the injury (Fong et al, 2009). In order to prevent ankle sprain injury, taping and bracing are commonly used by athletes (Cordova et al., 2007). However, these methods restrict the freedom of ankle joint motion and hence affect the performance in sports (Hume \& Gerrard, 1998). Recently, an intelligent sprain-free shoe that protects the ankle from spraining injury has been developed (Chan et al., 2006). It does not restrict ankle joint motion and allows freedom of movement in normal condition, meanwhile, it provides support and protects the athlete from injury when the ankle is in danger to sustain a supination ankle sprain. For this innovative design, an identification system has to be developed to monitor the ankle joint biomechanics and detect if there is an ankle sprain motion, and 
ultimately actuate the correction mechanism to protect the ankle joint from sustaining an injury.

Fong and his colleagues (2008) developed a three-pressure-sensor (3PS) system on insole to monitor the ankle supination torque for the abovementioned purpose. The system worked well in walking, running, cutting, vertical jump-landing and stepping-down motions. However, the development of the system did not involve ankle sprain motion, which could not be performed on a flat force plate in order to capture the essential kinetics data for ankle supination torque calculation. Therefore, we comment that while the previous system worked well as a mobile system to monitor ankle supination torque, it might not be applicable to estimate the joint torque and identify a spraining motion during a real injury event.

This paper presents an identification system to detect ankle supination sprain motion. Gyrometers and accelerometers were used for collecting dorsal foot kinematics data for training up a Support Vector Machine (SVM) which serves as an identification system, which has been verified to have $91.3 \%$ accuracy. The method has been previously applied to identify different human motions, such as close-eye activities 
(Hsieh et al., 2007), fall detection (Shi et al., 2009) and gait pattern of young and old people (Begg \& Kamruzzaman, 2005).

The identification method would contribute as an essential part of the sprain-free shoe as a new and innovative prophylactic apparel for sports.

\section{Methods}

\section{Data collection}

Six male subjects $($ age $=21.2 \pm 1.7 \mathrm{yr}$, height $=1.72 \pm 0.05 \mathrm{~m}$, body mass $=61.5 \pm$ $3.1 \mathrm{~kg}$, foot length $=255.3 \pm 10.6 \mathrm{~mm}$ ) were recruited from the athletic team of The Chinese University of Hong Kong. Subjects with previous ankle injury were excluded. Anterior drawer test and talar tilt test were performed on both feet of the subjects to ensure there was no abnormality for their ankles. The university ethics committee approved the study.

Each subject performed a total of 100 trials: 50 trials of simulated supination sprain motion (Figure 2) and 50 trials of non-sprain motion. Simulated supination sprain motions were performed on the supination sprain simulator (Chan et al., 2008) which simulated ankle spraining motion with different combinations of inversion and plantarflexion (I: total inversion / II: 23 degree supination / III: 45 degrees 
supination / IV: 67 degrees supination / V: total plantarflexion). These inversion and plantarflexion angles were chosen to allow a wide range of collected data. Each of the above inversion and plantarflexion angles contributed 10 trials respectively. The sequences of data collection at different angles were randomly assigned. Each subject placed their feet on the rotating disc of the supination sprain simulator with shoulder width apart. They were instructed to stand with weight evenly distributed on both feet. Either left or right platform fell in each trial randomly. Motion sensors were attached on the right feet only, and thus only the trials with right foot perturbation were collected. Ten trials of right foot perturbations were performed at each angle. Between each trial, subjects were allowed to have sufficient rest to prevent fatigue which was reported verbally by the subject. After collecting data for simulated supination sprain motion, non-sprain motions data were collected, including walking, running, cutting, stepping-down and vertical jump-landing. Each motion contributed 10 trials. These motions were chosen because they are common in human daily activities. The sequences of data collection of different non-sprain motions were random. In walking and running trials, subjects were requested to walk or run at their preferred speed for five consecutive strides. Data collection started from the first stride. For cutting trials, subjects were requested to run for five consecutive strides with full speed and perform a cutting motion of 90 degrees with 
their right limb. In stepping-down trials, subjects stepped down from a 30cm high step with their right limb landing first. For vertical jump-landing, subjects were requested to perform vertical jump-landing with both legs to their maximum height. Subjects were allowed to rest between each trial.

During data collection, eight wired motion sensors (Sengital Ltd., Hong Kong, China) were attached to the right foot at hallux, first proximal metatarsal head, fifth distal metatarsal head, fifth proximal metatarsal head, medial calcaneus, posterior calcaneus, lateral malleolus and tibia (Figure 1). The sensors were attached by the same research staff who has adequate knowledge to identify these anatomical landmarks throughout the study to ensure consistency. The size of the motion sensor was $20 \mathrm{~mm} \times 18 \mathrm{~mm} \times 6 \mathrm{~mm}$. Each motion sensor was then connected to a single printed circuit board (PCB) with a size of $50 \times 25 \times 15 \mathrm{~mm}$. The PCB was responsible for the communication between its dedicated sensor and the computer for data collection. Each motion sensor consists of a tri-axial accelerometer and gyrometer, collecting three-dimensional linear acceleration $\left(\mathrm{A}_{\mathrm{x}}, \mathrm{A}_{\mathrm{y}}, \mathrm{A}_{\mathrm{z}}\right)$ and three dimensional angular velocity $\left(G_{x}, G_{y}, G_{z}\right.$, ) at a sampling frequency of $500 \mathrm{~Hz}$. This sampling frequency is much higher when compared with the sensors being used in some other previous studies, ranging from 20 to $200 \mathrm{~Hz}$ (Bernmark \& Wiktorin, 2002; Coley et 
al., 2007), as to collect adequate data during vigorous sprain motions which happen within 50ms (Fong et al, 2009).

Support Vector Machine for classification of human motion

Support Vector Machine (SVM) is one of the techniques in statistical learning theory for classification. SVM was divided into two main parts: training and verification (Vapnik, 1995).

Training the Support Vector Machine

The learning theory of the Support Vector Machine can be expressed as a function $f: \Re^{n} \rightarrow \pm 1$ where $y=f(x)$. This function maps patterns $x$ to the classification $y$. The function $f(x)$ can be expressed as:

$$
f(x)=\sum_{i=1}^{N} y_{i} \alpha_{i} K\left(x, x_{i}\right)+b,
$$

where $N$ is the number of training patterns, $\left(x_{i}, y_{i}\right)$ is training pattern $i$ with its classification as $y_{i}, \alpha_{i}$ and $b$ are learned weights. $\mathrm{K}(\cdot)$ is a kernel function $K\left(\bar{x}_{i}, \bar{x}_{j}\right)=\Phi\left(\bar{x}_{\mathrm{i}}\right) \cdot \Phi\left(\overline{\mathrm{x}}_{\mathrm{j}}\right)$ (Cristianini \& Shawe-Taylor, 2000), which could be any symmetric kernel function that satisfies the Mercer's condition corresponding to a dot product in some feature space (Bernhard et al., 1998). $\left(x_{i}, y_{i}\right)$ with $\alpha_{i}>0$ are sets of support vectors. The surface $f(x)=0$ is a hyperplane through the 
feature space defined by the kernel function. Optimal parameters $\alpha_{i}$ and $b$ are selected to minimize the number of incorrect classifications by maximizing the distance of the support vectors to the hyperplane $f(x)=0, \quad y_{i}>0$ indicates a simulated supination sprain trial, where $y_{i}<\mathbf{0}$ indicates a non-sprain trial. Maximize:

$$
L_{D} \equiv \sum_{i=1}^{N} \alpha_{i}-\frac{1}{2} \sum_{i=1}^{N} \sum_{j=1}^{N} y_{i} y_{j} \alpha_{i} \alpha_{i} k\left(x_{i}, x_{i}\right)
$$

Subject to:

$0 \leq \alpha_{i} \leq c_{y} \quad \sum_{i=1}^{N} y_{i} \alpha_{i}=0$.

The constant $C$ denotes the penalty to errors, therefore it affects the tolerance to incorrect classifications. After solving the equation (2) and finding $\alpha_{i}$, we can use any other support vector $\left(x_{i}, y_{i}\right)$ to find $b$.

In the training process, a value of signal strength (unitless) of each of the eight motion sensors was calculated to quantify its capacity to identify the spraining and non-spraining motions. The signal strength ranges from 0 to 1024 , while higher signal strength means that the signal could better identify the two group of motions. SVM training was done with the data from the sensor of highest signal strength value. Six subjects contributed to 600 trials, including 300 simulated supination 
sprain and 300 non-sprain trials. For each trial, one second of data of all the six channels $\left(G_{x y}, G_{y y}, G_{z^{y}}, A_{x^{y}} A_{y^{y}} A_{z}\right)$ was trimmed. The data was then converted to frequency domain by discrete Fourier transform (DFT). This process allowed the waveform characteristics being extracted for the training. Only low frequency components were fed into the Support Vector Machine, while high frequency noises were eliminated. Similar procedure was adopted in previous studies of fall detection (Shi et al., 2009). The conversion was done by Matlab (version R2007a, MathWorks, Inc., Natick, Massachusetts, USA). The data in frequency domain was then used for the training process of the Support Vector Machine (Joachims, 1999). The Support Vector Machine then generated the model which consists of a hyperplane to separate the two sets of data: sprain motion and common sporting activities.

\section{Support Vector Machine verification}

Another six male subjects $($ age $=22.0 \pm 1.7 \mathrm{yr}$, height $=1.75 \pm 0.04 \mathrm{~m}$, body mass $=$ $69.7 \pm 2.8 \mathrm{~kg}$, foot length $=262.0 \pm 9.9 \mathrm{~mm}$ ) were recruited to perform the validation test. The same data collection procedure for the aforementioned SVM training session was done. Each subject performed a total of 100 trials: 50 trials of simulated supination sprain motion (Figure 2) and 50 trials of non-sprain motion. One second of sensor data from each trial was then trimmed and converted to 
frequency domain using DFT. Processed data was then fed into the trained SVM model. The accuracy was calculated by the percentage of trials being correctly identified. The trained SVM model was considered to be effective when the accuracy achieved 90\% (Lau et al., 2008). If the training is not successful, the training process would be performed again with the data from sensor of second highest signal strength. If the SVM training of using single sensor data was not successful, combinations of two or more sensors would be performed. This increased the number of support vectors chosen, and thus the accuracy of the identification method.

\section{Results}

The signal strength of each sensor was shown in Table 1. As the signal strength of medial calcaneus is the highest, the data obtained from sensor located at the medial calcaneus was selected to train up the SVM model. After training the SVM with the 600 trials of data from sensor located at the medial calcaneus, 521 support vectors, with threshold $b=0.46$, were selected to build the SVM model in equation (1) for classification.

The other 600 data were then fed to the SVM model for validation. Among the data, 
548 were correctly classified while 52 were classified incorrectly. The accuracy on the test set was $91.3 \%$, therefore successful identification was considered achieved. As the accuracy is higher than $90 \%$, the medial calcaneus was chosen as the sensor position.

The details of the verification result were shown in Table 2 and 3. Within the 300 simulated supination sprain trials, the SVM model could correctly identify 291 trials. Among the nine simulated supination sprain trials that cannot be classified correctly, eight of the trials are recorded when the fall platform angle was 90 degrees, while the other one trial was with platform angle set at 67 degrees. All the simulated supination sprain trials with platform angle at zero degree, 23 degrees, 45 degrees were identified correctly. For the 300 non-sprain trials, 43 trials were identified incorrectly as false alarm. Among the five non-sprain motions, stepping-down, vertical jump-landing contributed to $32.6 \%, 25.6 \%$ and $25.6 \%$ of false alarm respectively.

\section{Discussion}

From the result shown in Table 2, our developed method correctly identified $97.0 \%$ of the simulated ankle supination sprain motions. Our result is encouraging, as it is 
comparable to previous successful studies achieving 95-100\% accuracy in fall identification (Shi et al., 2009 and Bourke et al., 2007). Among the non-sprain motions, $14.3 \%$ false alarm was recorded - these common sporting motions were misidentified as hazardous. We comment that this is acceptable as the method as a whole is conservative - it provides unnecessary protection to $14.3 \%$ of the trials which does not need protection, and it fails to provide such protection to only $3 \%$ of the trials which needs protection.

In this research, one second of data was extracted from each trial. This time frame was chosen because it covered at least one cycle of motion in both the simulated supination motion and non-sprain motion and served as a good start to test the feasibility for training up the identification method. However, as real sprain motion occurs within $50 \mathrm{~ms}$, in order to serve as a real-time alarm system, it is essential to trim down the time duration, or the window size, but keep obtaining adequate data for the identification procedure with good accuracy. More data analysis work should be done in the future to find out the minimal and workable window size in order to catch up and actuate the corrective function of the proposed sprain-free shoe.

Within all the incorrectly identified simulated supination sprain trials, 8 out of 9 
were found when the platform angle was set at 90 degrees. When platform angles were set at 90 degrees, the ankle mainly underwent a plantarflexion motion which was common in normal many common sporting activities, such as running, walking, jumping and walking downstairs. Also, simulated supination sprain motion was in sub-injury level, which was less vigorous than real sprain, therefore some trials were misclassifying with platform angle set at 90 degrees.

The unit of the sensor value is arbitrary and has no actual physical meaning. It is not necessary to calibrate the sensor for the classification purpose because the sensor data was fed directly to train up the Support Vector Machine (SVM) and the same type of sensor were used in the validation stage. Filter such as Butterworth low pass filter and critically damped filter were commonly used in smoothing motion sensor data (Erer, 2007). However, we decided not to apply filter in this research because the waveform of the filtered data using a Butterworth low pass filter with $20 \mathrm{~Hz}$ generally showed no change when compare with the raw data. The simplified process utilizing unfiltered raw data is important, because it saved processing time and facilitated the development of a quick and real-time identification method.

All the supination sprain data were biomechanically simulated but not real sprain 
incidents, since performing real injury trials in laboratory is unethical and also not practical. We could only define them as sub-injury trials but not ligamentous sprain injury trials. Although these sub-injury trials were less vigorous, such motions are not undesirable in a real sporting situation as the ankle joint is approaching an un-returnable excessive supination orientation which may lead to ligamentous sprain injury. Besides, we believed that those simulated supination sprain motions are less vigorous than the real sprain motions. This means that the simulated sprain motion have measured linear acceleration and angular velocity more similar to that of common sporting motions. Thus, we believed that as this classification model can classify simulated sprain motion from common sporting motions in 91.3\% accuracy, the system can work at a similar or even better accuracy in real sprain classification. However, this belief can only be proved by manufacturing prototype which will be applied directly to athletics during training and competitions.

The identification method now included trials of five types of simulated supination sprain motions and five types of non-sprain common sporting sporting motions. It covered most of the common motions in daily life. Therefore the model is good for identification of ankle sprain motion during common sporting activities. However, it is also possible to develop some sport-specific ankle sprain detection system in the 
future to further increase the accuracy. This can be done by including some special movement of a specific sport during SVM training.

In this paper, a wired version of sprain identification method was developed. In the near future, we are going to develop a wireless version prototype. The SVM model can be built in on a PCB with DC power supply. The PCB can be located near the ankle, so that the system can become wireless. Real time recognition is possible in the future development by applying sliding window for data trimming. Sliding window allows real time trimming of data at different time point. However, the size of the window, therefore number of data required for classification of simulated supination motion, has to be further investigated. The final process time for real time classification of the system would mostly depend on the window size and also the performance of the hardware on the PCB.

\section{Conclusion}

This research introduces a method to classify sub-injury ankle supination sprain motions from non-sprain sporting motions using one motion sensor sampling at $500 \mathrm{~Hz}$ with $91.3 \%$ accuracy. Future work of the study includes minimize the window size, therefore the time frame for real time detection, this is important in 
reducing the processing time for classification. The sampling frequency of the motion sensors should be minimized, this can reduce the cost of sensors, which is currently US\$100 each. This can also minimize the amount of data to be processed. Hence, the time for data processing can be further reduced to make real time classification more feasible. In the near future, the whole system can be built with only one motion sensor at lower sampling frequency with a PCB with trained SVM model and DC power supply at a low cost.

\section{Acknowledgement}

The study was financially supported by the Innovation Technology Fund from the Innovation and Technology Commission, Hong Kong Special Administrative Region Government, Project Number: ITS/013/07. The authors acknowledge Dr Alan Lam and $\mathrm{Mr}$ Joe Wong of Sengital Limited to provide technical assistance in the fabrication of the motion sensors.

\section{$\underline{\text { References }}$}

Begg, R., Kamruzzaman, J. (2005). A machine learning approach for automated recognition of movement patterns using basic, kinetic and kinematic gait data. Journal of Biomechanics, 38(3), 401-408. 
Bernhard, S., Burges, C. J. C., Smola, A. (1998). Advanced in Kernal methods support vector learning.Cambridge, MA: MIT Press.

Bernmark, E., Wiktorin, C. (2002). A triaxial accelerometer for measuring arm movements. Applied Ergonomics, 33(6), 541-547.

Bouke, A.K., O’Brien J.V., Lyons G.M. (2007). Evaluation of a threshold-based tri-axial accelerometer fall detection algorithm. Gait and Posture, 26(2), 194-199.

Chan, K. M. (2006). Ankle injuries in sports - what's new on the horizon. Journal of Medical Biomechanics. 21(Supplement), 6-7.

Chan, Y. Y., Fong, D. T. P., Yung, P. S. H., Fung, K. Y., Chan, K. M. (2008). A mechanical supination sprain simulator for studying ankle supination sprain kinematics. Journal of Biomechanics. 41(11), 2571-2574.

Coley, B., Jolles, B. M., Farron, A., Bourgeois, A., Nussbaumer, F., Pichonnaz, C., et al. (2007). Outcome evaluation in shoulder surgery using 3D kinematics sensors. Gait and Posture, 25(4), 523-532.

Cordova, M. L., Dorrough, J. L., Kious, K., Ingersoll, C. D., Merrick, M. A. (2007). Prophylactic ankle bracing reduces rearfoot motion during sudden inversion. Scandinavian Journal of Medicine and Science in Sports, 17(3), 216-222.

Cristianini, N., Shawe-Taylor, K. (2000). An introduction to support vector 
machines.Cambridge, UK: Cambirdge University Press.

Erer, K. S. (2007). Adaptive usage of the butterworth digital filter. Journal of Biomechanics, 40(13), 2934-2943.

Fong, D. T. P., Hong, Y., Chan, L. K., Yung, P. S. H., Chan, K. M. (2007). A systematic review on ankle injury and ankle sprain in sports. Sports Medicine, 37(1), 73-94.

Fong, D. T. P., Chan, Y. Y., Hong, Y., Yung, P. S. H., Fung, K. Y., Chan, K. M. (2008). A three-pressure-sensor (3PS) system for monitoring ankle supination torque during sport motions. Journal of Biomechanics, 41(11), 2562-2566.

Fong, D. T. P., Chan, Y. Y., Mok, K. M., Yung, P. S. H., Chan, K. M. (2009). Understanding acute ankle ligamentous sprain injury in sports. Sports Medicine, Arthroscopy Rehabilitation, Therapy \& Technology, 1:14.

Hsieh, C. W., Kan, C. W., \& Jong, T. L. (2007). Analysis of closed eyes motion using a wireless eye-mask. Medical and Biological Engineering \& Computing, $45,365-374$.

Hume, P. A., Gerrard, D. F. (1998). Effectiveness of external ankle support. Bracing and taping in rugby union. Sports Medicine, 25(5), 285-312.

Joachims, T. (1999). Making large-scale SVM learning practical. Advances in lernel methods - support vector learning:MIT Press. 
Lau, H. Y., Tong, K. Y., Zhu, H. (2008). Support vector machine for classification of walking conditions using miniature kinematic sensors. Medical and Biological Engineering and Computing, 46(6), 563-573.

Shi, G., Chan, C. S., Li, W.J., Leung, K.S., Zou, Y., Jin, Y. (2009). Mobile human airbag system for fall protection using MEMS sensors and embedded SVM classifier. IEEE Sensors Journal, 9(5), 495-509.

Vapnik, V. (1995). The nature of satistical learning theory. New York: Springer-Verlag. 
Figure 1 - Eight motion sensors attached on the right foot

Figure 2 - Experimental setup for data collection on ankle simulated sprain. 
Click here to download high resolution image

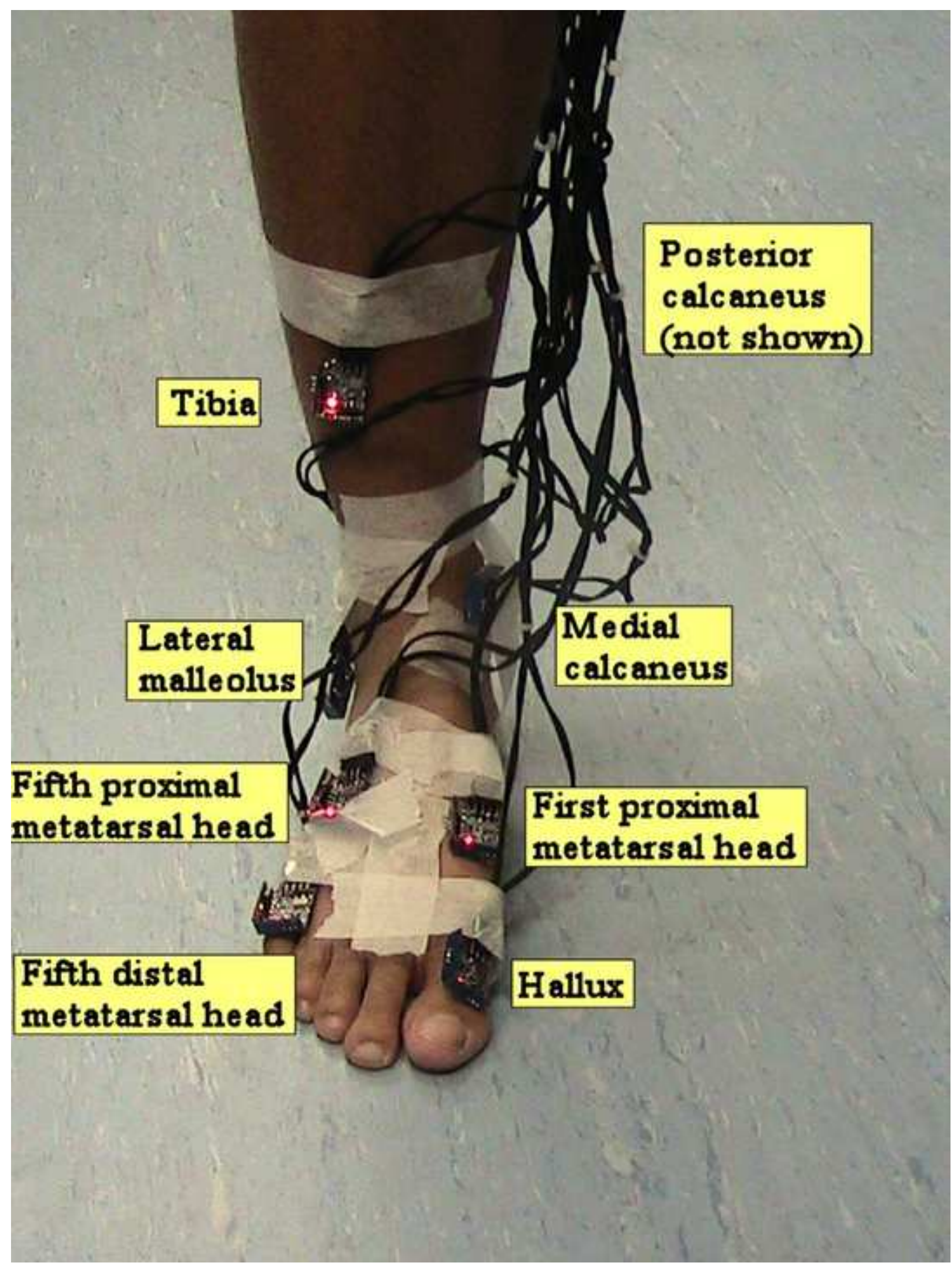


Figure 2
Click here to download high resolution image

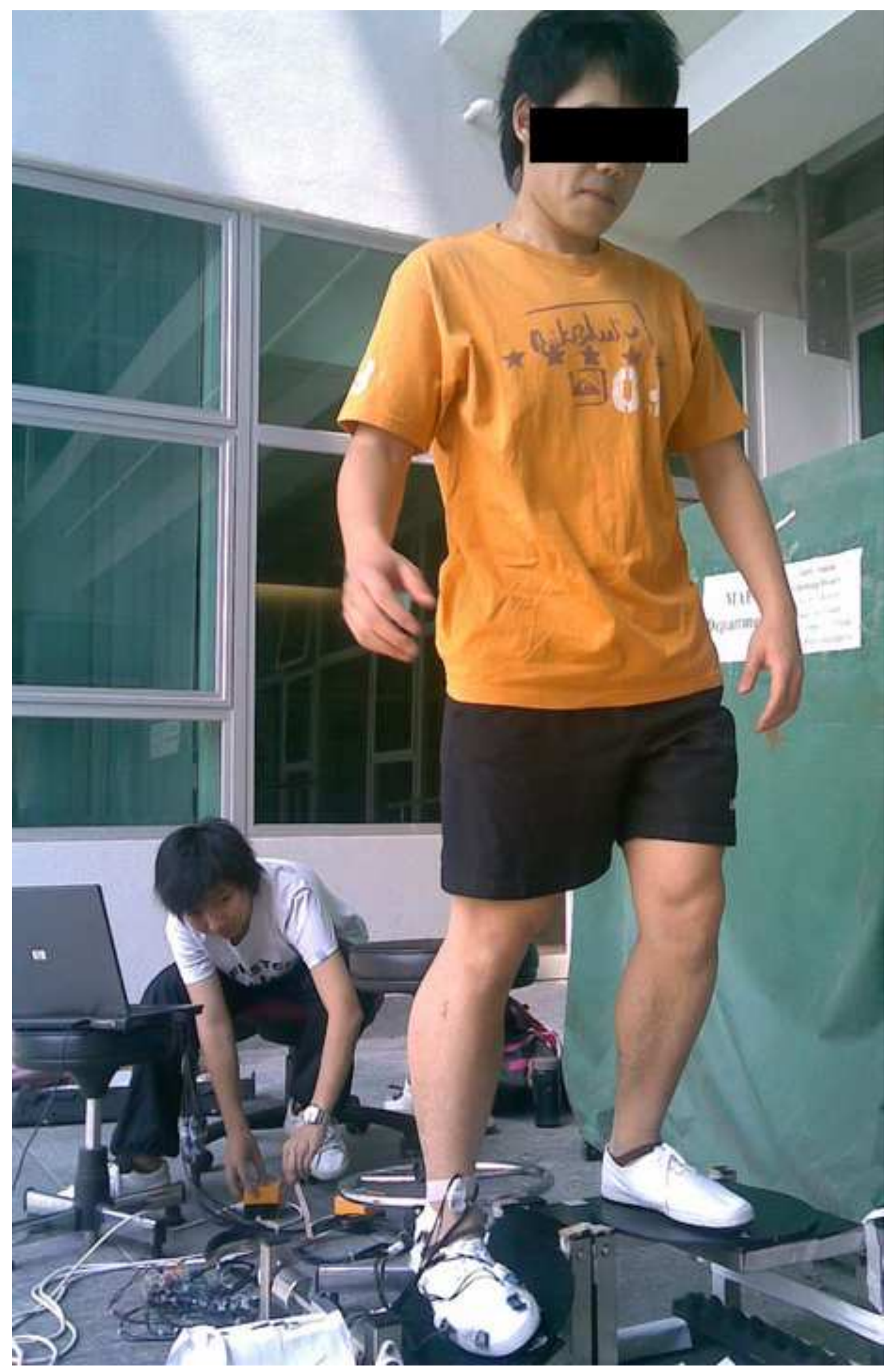


Table 1 - Signal strength at different sensor position

\begin{tabular}{lc}
\hline \multicolumn{1}{c}{ Sensor position } & Signal strength $^{\#}$ (Standard deviation)(unitless) \\
\hline Hallux & $431.6(9.4)$ \\
First proximal metatarsal head & $436.9(10.9)$ \\
Fifth distal metatarsal head & $418.7(12.7)$ \\
Fifth proximal metatarsal head & $381.0(10.6)$ \\
Medial calcaneus & $464.1(11.8)$ \\
Posterior calcaneus & $426.9(10.1)$ \\
Lateral malleolus & $417.7(12.1)$ \\
Tibia & $431.6(10.7)$ \\
\hline
\end{tabular}

${ }^{\#}$ Signal strength ranges from 0 (weakest) - 1024 (strongest)

Table 2 - Results of verification of the Support Vector Machine model.

\begin{tabular}{ccc}
\hline & $\begin{array}{c}\text { Number of correctly } \\
\text { identified trials }\end{array}$ & $\begin{array}{c}\text { Number of incorrectly } \\
\text { identified trials }\end{array}$ \\
\hline Simulated supination sprain trials & $291(97.0 \%)$ & $9(3.0 \%)$ \\
Common sporting trials & $257(85.7 \%)$ & $43(14.3 \%)$ \\
\hline
\end{tabular}


Table 3 - Number of incorrect identified trials during the verification process.

\begin{tabular}{|c|c|c|c|c|c|c|c|c|c|}
\hline & & $\# 1$ & $\# 2$ & $\# 3$ & $\# 4$ & $\# 5$ & \#6 & Sub-total & Total \\
\hline \multirow{5}{*}{$\begin{array}{c}\text { Simulated } \\
\text { supination } \\
\text { sprain trials }\end{array}$} & 0 degree & - & - & - & - & - & - & 0 & \multirow{5}{*}{9} \\
\hline & 23 degrees & - & - & - & - & - & - & 0 & \\
\hline & 45 degrees & - & - & - & - & - & - & 0 & \\
\hline & 67 degrees & - & - & - & 1 & - & - & 1 & \\
\hline & 90 degrees & - & - & 2 & 5 & 1 & - & 8 & \\
\hline \multirow{5}{*}{$\begin{array}{c}\text { Common } \\
\text { sporting trials }\end{array}$} & Cutting & - & 1 & 2 & 2 & - & 6 & 11 & \multirow{5}{*}{43} \\
\hline & Stepping-down & - & 6 & - & 5 & - & 3 & 14 & \\
\hline & $\begin{array}{c}\text { Vertical } \\
\text { jump-landing }\end{array}$ & - & 7 & 4 & - & - & - & 11 & \\
\hline & Running & - & - & 2 & - & - & - & 2 & \\
\hline & Walking & - & 5 & - & - & - & - & 5 & \\
\hline
\end{tabular}




\section{Conflict of interest}

Editor of Journal of Biomechanics,

REF: Submission of manuscript "Identification of Ankle Sprain Motion from Normal Activities by Dorsal Foot Kinematics Data"

The authors declare no financial and personal relationships with other people or organizations that could inappropriately influence this submitted work.

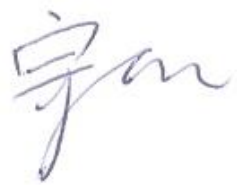

Yue-Yan CHAN

November 4th, 2009. 